\title{
POWERSUM FORMULA FOR DIFFERENTIAL RESOLVENTS
}

\author{
JOHN MICHAEL NAHAY
}

Received 5 October 2002

\begin{abstract}
We will prove that we can specialize the indeterminate $\alpha$ in a linear differential $\alpha$-resolvent of a univariate polynomial over a differential field of characteristic zero to an integer $q$ to obtain a $q$-resolvent. We use this idea to obtain a formula, known as the powersum formula, for the terms of the $\alpha$-resolvent. Finally, we use the powersum formula to rediscover Cockle's differential resolvent of a cubic trinomial.
\end{abstract}

2000 Mathematics Subject Classification: 12H05, 13 N15.

1. Introduction. It was proved in [4, Theorem 37, page 67] that for any integer $q$, a polynomial $P(t) \equiv \sum_{k=0}^{N}(-1)^{N-k} e_{N-k} t^{k}$ of a single variable $t$ whose coefficients $\left\{e_{N-k}\right\}_{k=0}^{N}$ lie in an ordinary differential ring $\mathbb{R}$ with derivation $D$ possesses an ordinary linear differential $\alpha$-resolvent and an ordinary linear differential $q$-resolvent, where $\alpha$ is a constant, transcendental over $\mathbb{R}$. With no loss of generality, we assume that $P$ is monic and has no zero roots. Then the coefficient $e_{N-k}$ is the $(N-k)$ th elementary symmetric function of the roots of $P$. We assume that $P(t)=\prod_{k=1}^{n}\left(t-z_{k}\right)^{\pi_{k}}$ has $n \leq N$ distinct roots and $\pi_{k}$ is the multiplicity of the root $z_{k}$ in $P$. It was proved in [2] that for each root $z_{k}$, there exists a nonzero solution $y_{k}$ of the logarithmic differential equation $D y_{k} / y_{k}=\alpha \cdot\left(D z_{k} / z_{k}\right)$. Obviously, such solutions are unique only up to a constant multiple. We define the notation $z_{k}^{\alpha}$ to represent any such solution $y_{k}$. Hence, we will call $y_{k}$ an $\alpha$-power of $z_{k}$. From now on, we will drop the subscript $k$ on $z_{k}$ and $y_{k}$. It will be understood that a different $z$ implies a different $y$.

In this paper, we present the powersum formula as a new method for computing resolvents, although it remains a conjecture whether the powersum formula always yields a (nonzero) resolvent rather than an identically zero equation. It was proved in [5, Theorem 4.1, page 726] that if all the distinct roots of a polynomial are differentially independent over constants, then the powersum formula yields a resolvent. It was shown in [6, Section 11, pages 344-345], how the solution of the Riccati nonlinear differential equation is related to the resolvent of a quadratic polynomial.

2. Notation. Let $\mathbb{N}$ denote the set of positive integers. Let $\mathbb{N}_{0}$ denote the set of nonnegative integers. Let $\mathbb{Z}^{\#}$ denote the set of nonzero integers. The following notation has been slightly modified from Kolchin's notation in [2] and Macdonald's notation in [3]. Let $\mathbb{Z}\{e\}$ denote the differential ring generated by the integers $\mathbb{Z}$ and the $N$ coefficients $e \equiv\left\{e_{k}\right\}_{k=1}^{N}$ of $t$ in $P$. Let $\mathbb{Q}\langle e\rangle$ denote the differential field generated by the rational numbers $\mathbb{Q}$ and $e$. For each $m \in \mathbb{N}$, let $\mathbb{Z}\{e\}_{m}$ denote the ordinary (nondifferential) ring 
generated by $\mathbb{Z}, e$, and the first $m$ derivatives of $e$. (A differential ring must contain infinitely many derivatives of any of its elements.) Let $\mathbb{Q}\langle e\rangle_{m}(z)=\mathbb{Q}\langle e\rangle_{m}[z]$ denote the field generated by $\mathbb{Q}, e$, the first $m$ derivatives of $e$, and the single root $z$. From this point on, we will write $\mathbb{Q}\langle e\rangle_{m}[z]$ instead of $\mathbb{Q}\langle e\rangle_{m}(z)$ for this field to emphasize the fact that elements in this field are polynomial in the root $z$. If $\mathbb{R}$ represents any of the rings or fields mentioned so far, then let $\mathbb{R}[t, \alpha]$ denote the polynomial ring in the indeterminates $t$ and $\alpha$ over $\mathbb{R}$.

Let $\theta \equiv(n !) \cdot\left(\prod_{k=1}^{n} \pi_{k}\right) \cdot\left(\prod_{k=1}^{n} z_{k}\right) \cdot\left(\prod_{i<j}\left(z_{i}-z_{j}\right)^{2}\right)$. By our conditions on $P, \theta \neq 0$. It is also easy to show that $\theta \in \mathbb{Z}[e]$. For each $m \in \mathbb{N}$, it was proved in $[4$, Theorem 32 , page 60] that there exists a polynomial $G_{m}(t, \alpha)$ in $t$ and $\alpha$ satisfying the following definition.

DEFINITION 2.1. Define $G_{m}(t, \alpha)$ to be the polynomial in $t$ and $\alpha$ such that $G_{m}(z, \alpha)$ $=D^{m} y /(\alpha \cdot y)$ for each root $z$ of $P$ and $\theta^{m} \cdot G_{m}(t, \alpha) \in \mathbb{Z}\{e\}_{m}[t, \alpha]$.

A specialization $\phi$ is a ring homomorphism $\phi: \mathbb{R} \rightarrow \mathbb{R}$ from a ring $\mathbb{R}$ into an integral domain $\hat{\mathbb{R}}$. For any polynomial $P(t)=\sum_{k=0}^{N}(-1)^{N-k} e_{N-k} \cdot t^{k} \in \mathbb{R}[t], \phi(P)$ is defined to be the polynomial $(\phi P)(t)=\sum_{k=0}^{N}(-1)^{N-k} \phi\left(e_{N-k}\right) \cdot t^{k} \in \mathbb{R}[t]$. A differential specialization $\phi$ is a specialization $\phi: \mathbb{R} \rightarrow \hat{\mathbb{R}}$ from a differential ring $\mathbb{R}$ with derivation $D$ into a differential integral domain $\hat{\mathbb{R}}$ with derivation $\hat{D}$ such that $\phi D=\hat{D} \phi$ on $\mathbb{R}$.

3. Specializing $\alpha$. Let $q \in \mathbb{N}$. Let $\phi_{q}: \mathbb{Q}\langle e\rangle[z, \alpha] \rightarrow \mathbb{Q}\langle e\rangle[z]$ be the ring specialization such that $\phi_{q}$ is the identity on $\mathbb{Q}\langle e\rangle[z]$ and $\phi_{q}(\alpha)=q$. We may compute $D z^{q} /\left(q \cdot z^{q}\right)$. Since $\phi_{q}$ is not defined to act on $y$, we are not able to specialize $y$ to $z^{q}$ in Theorem 3.1. However, $\phi_{q}$ is defined to act on $D^{m} y /(\alpha \cdot y)$ since $D^{m} y /(\alpha \cdot y)=G_{m}(z, \alpha) \in \theta^{-m}$. $\mathbb{Z}\{e\}_{m}[z, \alpha]$. Theorem 3.1 asserts that $G_{m}(z, q)=D^{m} z^{q} /\left(q \cdot z^{q}\right)$. Theorem 3.2 asserts that $\phi_{q}\left(D^{m} y /(\alpha \cdot y)\right)=D^{m} z^{q} /\left(q \cdot z^{q}\right)$.

THEOREM 3.1. Assume all the same definitions and notations as in the introduction. Then the mth derivative of $z^{q}$ can be expressed as a product of $q \cdot z^{q}$ and an element in $\mathbb{Q}\langle e\rangle_{m}[z]$. More specifically, $G_{m}(z, q)=D^{m} z^{q} /\left(q \cdot z^{q}\right)$, where $G_{m}(z, q) \in \mathbb{Q}\langle e\rangle_{m}[z]$ and $G_{m}(t, \alpha)$ was given in Definition 2.1.

Proof. For brevity, write $G_{m}=G_{m}(z, \alpha)$ for the particular root $z$. We emphasize that $G_{m}$ is $G_{m}(t, \alpha)$ with $t$ specialized to the particular root $z$. We find that $\theta \cdot\left(D z^{q} /(q\right.$. $\left.\left.z^{q}\right)\right)=\theta \cdot(D z / z)=\theta \cdot G_{1} \in \mathbb{Z}\{e\}_{1}[z]$. Therefore,

$$
\begin{aligned}
D z^{q} & =q \cdot z^{q} \cdot G_{1} \Longrightarrow D^{2} z^{q}=q \cdot\left(q \cdot z^{q-1} D z \cdot G_{1}+z^{q} \cdot D G_{1}\right) \\
& =q \cdot z^{q}\left(q \cdot\left(\frac{D z}{z}\right) \cdot G_{1}+D G_{1}\right)=q \cdot z^{q}\left(q \cdot G_{1}^{2}+D G_{1}\right) \\
& =q \cdot z^{q} \cdot \phi_{q}\left(\alpha \cdot G_{1}^{2}+D G_{1}\right)=q \cdot z^{q} \cdot \phi_{q}\left(G_{2}\right) .
\end{aligned}
$$

So, $D^{m} z^{q}=q \cdot z^{q} \cdot \phi_{q}\left(G_{m}\right)$ is true for $m=1$. Now assume that it is true for $m \geq 2$. Then $D^{m+1} z^{q}=q \cdot\left(q \cdot z^{q-1}(D z) \cdot \phi_{q}\left(G_{m}\right)+z^{q} \cdot D\left(\phi_{q}\left(G_{m}\right)\right)\right)$. But $\phi_{q}$ specializes $\alpha$, whose derivative is 0 , to an integer whose derivative is 0 . Thus, $D\left(\phi_{q}\left(G_{m}\right)\right)=\phi_{q}\left(D\left(G_{m}\right)\right)$. 
Hence,

$$
\begin{aligned}
D^{m+1} z^{q} & =q \cdot\left(q \cdot z^{q-1} \cdot(D z) \cdot \phi_{q}\left(G_{m}\right)+z^{q} \cdot \phi_{q}\left(D\left(G_{m}\right)\right)\right) \\
& =q \cdot z^{q} \cdot\left(q \cdot \frac{D z}{z} \cdot \phi_{q}\left(G_{m}\right)+\phi_{q}\left(D\left(G_{m}\right)\right)\right) \\
& =q \cdot z^{q} \cdot\left(\phi_{q}(\alpha) \cdot G_{1} \cdot \phi_{q}\left(G_{m}\right)+\phi_{q}\left(D\left(G_{m}\right)\right)\right) \\
& =q \cdot z^{q} \cdot \phi_{q}\left(\alpha \cdot G_{1} \cdot G_{m}+D\left(G_{m}\right)\right) \\
& =q \cdot z^{q} \cdot \phi_{q}\left(G_{m+1}\right) .
\end{aligned}
$$

Therefore, $D^{m+1} z^{q}=q \cdot z^{q} \cdot G_{m+1}(z, q)$ since $\phi_{q}$ affects only $\alpha$. By the principle of mathematical induction, this equation is true for all positive integers $m$.

Just because $D y /(\alpha \cdot y)=D z / z=D z^{q} /\left(q \cdot z^{q}\right)$ implies that $D y /(\alpha \cdot y)$ is independent of $\alpha$, it does not follow that $D^{m} y /(\alpha \cdot y)$ is independent of $\alpha$ for $m \geq 2$. We can see this by observing that $D^{m} y /(\alpha \cdot y) \neq D^{m} z / z \neq D^{m} z^{q} /\left(q \cdot z^{q}\right) \neq D^{m} y /(\alpha \cdot y)$ for $m \geq 2$.

THEOREM 3.2. Assume all the same definitions and notations as in Theorem 3.1 and Section 2. Then, for each $m \in \mathbb{N}$, the specialization under $\phi_{q}$ of $D^{m} y /(\alpha \cdot y)$ is $D^{m} z^{q} /(q$. $\left.z^{q}\right)$. That is, $\phi_{q}\left(D^{m} y /(\alpha \cdot y)\right)=D^{m} z^{q} /\left(q \cdot z^{q}\right)$.

Proof. By Definition 2.1, $D^{m} y /(\alpha \cdot y)=G_{m}(z, \alpha)$. By Theorem 3.1, $D^{m} z^{q} /\left(q \cdot z^{q}\right)=$ $G_{m}(z, q)$. Putting these results together yields

$$
\phi_{q}\left(\frac{D^{m} y}{\alpha \cdot y}\right)=\phi_{q}\left(G_{m}(z, \alpha)\right)=G_{m}(z, q)=\frac{D^{m} z^{q}}{q \cdot z^{q}} .
$$

4. Powersum satisfaction theorem and formula. An $\alpha$-resolvent of a polynomial $P(t) \equiv \sum_{i=0}^{N}(-1)^{n-i} e_{N-i} t^{i} \in \mathbb{F}[t]$ over a differential field $\mathbb{E}$ with derivation $D$ is a linear ordinary differential equation $\sum_{m=0}^{o} B_{m}(\alpha) \cdot D^{m} y=0$ of finite order $o$ such that each of the coefficient functions $B_{m}(\alpha)$ lies in the field $\mathbb{Q}\langle e\rangle(\alpha)$ (or preferably in the ring $\mathbb{Z}\{e\}[\alpha])$ such that not all $B_{m}(\alpha)$ are identically zero, and which is satisfied by the $\alpha$ power of every root $z$ of $P$. In other words, the coefficient functions of the resolvent are independent of the choice of root and are not all zero. By [4, Theorem 37, page 67], resolvents for any polynomial are guaranteed to exist. We state this assertion in Theorem 4.1.

THEOREM 4.1. Let $P(t) \equiv \sum_{i=0}^{N}(-1)^{n-i} e_{N-i} t^{i} \in \mathbb{F}[t]$ be a polynomial of degree $N$ in $t$ over a d-field $\mathbb{F}$ with $n$ distinct roots $\left\{z_{i}\right\}_{i=1}^{n}$. Then there exists an oth order differential resolvent $\sum_{m=0}^{o} B_{m}(\alpha) \cdot D^{m} y=0$ with $B_{m}(\alpha) \in \mathbb{Z}\{e\}_{n}[\alpha], B_{0}(0)=0$, and $\operatorname{deg}_{\alpha} B_{m}(\alpha) \leq$ $o(o-1) / 2-m+1$ for some $o \in[n]$. Furthermore, o may be chosen to equal the number of $\left\{y_{j}\right\}_{j=1}^{n}$ linearly independent over constants, and all solutions of this resolvent are linear combinations over constants of these o $y_{j}$ 's.

Theorem 4.1 gives us an upper bound on the degree in $\alpha$ in an $\alpha$-resolvent of $P$. Theorem 4.2 allows us to specialize the indeterminate $\alpha$ to an integer $q$ (or any number) to obtain a $q$-resolvent. 
THEOREM 4.2 (powersum satisfaction theorem). Let $P \in \mathbb{F}[t]$ be a monic polynomial with $n$ distinct roots $z=\left\{z_{i}\right\}_{i=1}^{n}$, none of which is zero and not all of which are constants. Let $q \in \mathbb{Z}$. If $R_{\alpha} \equiv \sum_{m=0}^{o} B_{m}(\alpha) \cdot D^{m} y$ is an $\alpha$-resolvent for $P$ of arbitrary order $o$, where $B_{m}(\alpha)=\sum_{i \geq 0} b_{i, m} \alpha^{i} \in \mathbb{Z}\{e\}[\alpha]$, with $b_{i, m} \in \mathbb{Z}\{e\}$, then $R_{\alpha}$ specializes to the $q$-resolvent $R_{q} \equiv \sum_{m=0}^{o} B_{m}(q) \cdot D^{m} y$ for $q \in \mathbb{Z}^{\#}$ under $\phi_{q}(\alpha)=q$ and $\phi_{q}(u)=u$ for each $u \in \mathbb{Z}\{e\}$. Furthermore, the qth powersum $p_{q}$ satisfies $\sum_{m=0}^{o} B_{m}(q) \cdot D^{m} p_{q}=0$ for each $q \in \mathbb{Z}^{\#}$.

Proof. By Definition 2.1 of $G_{m}(t, \alpha)$, we have

$$
\begin{aligned}
\sum_{m=0}^{o} B_{m}(\alpha) \cdot D^{m} y=0 & \Longleftrightarrow \sum_{m=0}^{o} B_{m}(\alpha) \cdot \frac{D^{m} y}{\alpha \cdot y}=0 \\
& \Longleftrightarrow \sum_{m=0}^{o} B_{m}(\alpha) \cdot G_{m}(z, \alpha)=0 .
\end{aligned}
$$

Now for each $q \in \mathbb{Z}^{\#}$, specialize this equation under $\phi_{q}$ to get $\sum_{m=0}^{o} B_{m}(q) \cdot G_{m}(z, q)=0$ by Theorem 3.2, since $\left.\phi_{q}\right|_{\mathbb{F}}=I$. For any of the roots of $P$, we have $G_{m}(z, q)=D^{m} z^{q}$ / $\left(q \cdot z^{q}\right)$ by Theorem 3.1. Thus,

$$
\sum_{m=0}^{o} B_{m}(q) \cdot \frac{D^{m} z^{q}}{q \cdot z^{q}}=0 \Longleftrightarrow \sum_{m=0}^{o} B_{m}(q) \cdot D^{m} z^{q}=0
$$

for each $q \in \mathbb{Z}^{\#}$. Therefore, an $\alpha$-resolvent specializes to a $q$-resolvent for each $q \in$ $\mathbb{Z}^{\#}$ under $\phi_{q}$. Now sum over the $N$ roots of $P$ including their multiplicities to get $\sum_{m=0}^{o} B_{m}(q) \cdot D^{m} p_{q}=0$ for each $q \in \mathbb{Z}^{\#}$.

The powersum satisfaction theorem states that for any monic polynomial $P$, the coefficients $b_{i, m}$ of $\alpha$ in any $\alpha$-resolvent $R_{\alpha} \equiv \sum_{(i, m) \in S} b_{i, m} \cdot \alpha^{i} D^{m} y$ of $P$ satisfy an infinite system of homogeneous equations

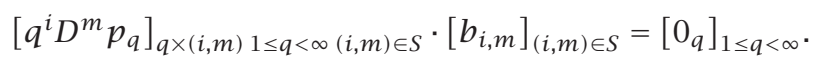

Here, $S$ denotes the set of pairs $(i, m)$ consisting of a power of $\alpha$, denoted by $i$, and an order of a derivative, denoted by $m$, such that $b_{i, m} \neq 0$. Let $|S|$ denote the size of $S$. We will be interested in proving that the rank

$$
\operatorname{rk}\left[q^{i} D^{m} p_{q}\right]_{q \times(i, m)} 1 \leq q<\infty(i, m) \in S
$$

of the matrix $\left[q^{i} D^{m} p_{q}\right]_{q \times(i, m)} 1 \leq q<\infty(i, m) \in S$ equals $|S|-1$ under certain circumstances. Under those circumstances, one can solve this system of equations to get a nonzero solution for $b_{i, m}$. The solution is given by $b_{i, m}=F_{i, m} \equiv(-1)^{\operatorname{sgn}(i, m)} \cdot\left|q^{i^{\prime}} D^{m^{\prime}} p_{q}\right|_{\left(i^{\prime}, m^{\prime}\right) \neq(i, m) q \in \Gamma}$, where $\operatorname{sgn}(i, m)$ indicates the ordering of the term $b_{i, m}$ in the resolvent, and we take $\Gamma$ to be the smallest possible set of positive integers that will guarantee a nonzero solution. In numerous examples, it has been found that $\Gamma \equiv\{k \in \mathbb{N} \ni 1 \leq k \leq|S|-1\}$. We call this the powersum formula for a resolvent of $P$. We use the notation $F_{i, m}$ to denote the terms of the resolvent obtained by this method to suggest the word formula. We will denote the resolvent obtained by this formula by $\mathfrak{R}_{\alpha}$. So, $\mathfrak{R}_{\alpha}=\left\{F_{i, m}\right\}$. 
If $\operatorname{rk}\left[q^{i} D^{m} p_{q}\right]_{q \times(i, m)} 1 \leq q<\infty(i, m) \in S=|S|$, then the only solution would be $b_{i, m}=0$ for all $(i, m) \in S$, contradicting the hypothesis that $R_{\alpha}$ is nonzero. Unfortunately, for a given polynomial $P$, one does not know a priori what the set $S$ of nonzero $b_{i, m}$ is or how large it is. Nevertheless, we may summarize the results obtained so far in a corollary to the powersum satisfaction theorem.

COROLlary 4.3 (the powersum formula). Let $R_{\alpha} \equiv \sum_{(i, m) \in S} b_{i, m} \cdot \alpha^{i} D^{m} y$ be an $\alpha$ resolvent of $P$, where $S \subset \mathbb{N}_{0} \times \mathbb{N}_{0}$ is a finite set. If there exists a set of $|S|-1$ integers $\Gamma \subset \mathbb{N}$ such that not all the $F_{i, m}$ given by the powersum formula $F_{i, m} \equiv(-1)^{\operatorname{sgn}(i, m)}$. $\left|q^{i^{\prime}} D^{m^{\prime}} p_{q}\right|_{\left(i^{\prime}, m^{\prime}\right) \neq(i, m)} q \in \Gamma$ are zero, then the linear ordinary differential equation (ODE), $\mathfrak{R}_{\alpha} \equiv \sum_{(i, m) \in S} F_{i, m} \cdot \alpha^{i} D^{m} y$, is an integral $\alpha$-resolvent of $P$. If no such set of integers $\Gamma$ exists, then the powersum formula yields all zeroes for $F_{i, m}$.

The author believes that the resolvent $\mathfrak{R}_{\alpha}$ given by the powersum formula will be a $\mathbb{Q}\langle e\rangle$-multiple, not just a $\mathbb{Q}\langle e\rangle(\alpha)$-multiple of $R_{\alpha}$, but this requires proof. For example, let $\alpha^{M} \cdot D^{H} y$ denote the highest power of $\alpha$ on the highest derivative of $y$ in $R_{\alpha}$. Even though $F_{M, L} / b_{M, L} \cdot R_{\alpha}$ and $\mathfrak{R}_{\alpha}$ are both resolvents (provided that $F_{M, L} \neq 0$ ) with the same coefficient function of $\alpha^{M} \cdot D^{H} y$, one must eliminate the possibility that their other terms may differ due to the possibility that $P$ has resolvents of lower order.

5. Example. We will now apply the powersum formula to compute a particular $\alpha$ resolvent of a particular trinomial. It has not yet been proved that this formula yields a nonzero differential equation for every polynomial. However, in every polynomial the author has tested, it has been possible to set up an $\alpha$-resolvent, itself a polynomial in the power $\alpha$, and choose the proper set of powersums such that the powersum formula yields a nonzero answer. If the powersum formula yields a nonzero answer, then it is guaranteed by Corollary 4.3 that the answer is a (nonzero) resolvent of the polynomial. By a very long and difficult proof in [4, Theorem 41, page 74] and [5, Theorem 4.1, page 726], it has been shown that in case the distinct roots of the polynomial are differentially independent over constants (i.e., they satisfy no polynomial differential equations over $\mathbb{Q})$, then the powersum formula yields a nonzero resolvent.

The powersum formula has the advantage of giving a resolvent in an integral form. In the next example, this means the powersum formula gives a resolvent all of whose terms lie in the ring $\mathbb{Z}[x, \alpha]$.

EXAMPLE 5.1 (Sir James Cockle's resolvent of a trinomial). Cockle [1] gave a formula for a linear differential $\alpha$-resolvent (although he did not call it that) for any trinomial of the form $t^{n}+x \cdot t^{p}-1$, where $D x \equiv 1$. Consider the particular trinomial $P(t) \equiv$ $t^{3}+x \cdot t^{2}-1$, where $n=3$ and $p=2$. Then, Cockle's resolvent specializes to $27 \cdot D^{3} y=$ $4 \cdot(x \cdot D+\alpha / 2)(x \cdot D+3 / 2+\alpha / 2)(x \cdot D-\alpha) y$. This expands to $27 \cdot D^{3} y=\left(4 \cdot(x \cdot D)^{3}+\right.$ $\left.6 \cdot(x \cdot D)^{2}-3 \cdot \alpha \cdot(1+\alpha) \cdot(x \cdot D)-\alpha^{2} \cdot(3+\alpha)\right) y$. Replacing $(x \cdot D)^{3}$ with $x^{3} \cdot D^{3}+3 \cdot x^{2}$. $D^{2}+x \cdot D$ and $(x \cdot D)^{2}$ with $x^{2} \cdot D^{2}+x \cdot D$ yields $\left(4 x^{3}-27\right) \cdot D^{3} y+18 \cdot x^{2} \cdot D^{2} y+(10-3 \cdot$ $\left.\alpha-3 \cdot \alpha^{2}\right) \cdot x \cdot D y-\alpha^{2} \cdot(3+\alpha) \cdot y=0$, which has the form $f_{1} \cdot D^{3} y+f_{2} \cdot D^{2} y+\left(f_{3}+f_{4}\right.$. $\left.\alpha+f_{5} \cdot \alpha^{2}\right) \cdot D y+\left(f_{6} \cdot \alpha^{2}+f_{7} \cdot \alpha^{3}\right) \cdot y=0$. The powersum formula requires one to know a priori the various powers of $\alpha$ appearing in a resolvent. Specialize $\alpha$ to one of the six integers $q \in\{1,2,3,4,5,6\}$, then sum the resulting equation over each of the three 
roots. Doing this for each $q \in\{1,2,3,4,5,6\}$, one gets a system of six linear equations in the undetermined coefficient functions $\left\{f_{k}\right\}_{k=1}^{7}$ of the form $2 \mathfrak{b} \cdot \vec{f}=\overrightarrow{0}$, where $2 \mathfrak{b}$ is the $6 \times 7$ matrix defined by

$$
\mathfrak{W} \equiv\left[\begin{array}{lllllll}
D^{3} p_{1} & D^{2} p_{1} & D p_{1} & 1 \cdot D p_{1} & 1^{2} \cdot D p_{1} & 1^{2} \cdot p_{1} & 1^{3} \cdot p_{1} \\
D^{3} p_{2} & D^{2} p_{2} & D p_{2} & 2 \cdot D p_{2} & 2^{2} \cdot D p_{2} & 2^{2} \cdot p_{2} & 2^{3} \cdot p_{2} \\
D^{3} p_{3} & D^{2} p_{3} & D p_{3} & 3 \cdot D p_{3} & 3^{2} \cdot D p_{3} & 3^{2} \cdot p_{3} & 3^{3} \cdot p_{3} \\
D^{3} p_{4} & D^{2} p_{4} & D p_{4} & 4 \cdot D p_{4} & 4^{2} \cdot D p_{4} & 4^{2} \cdot p_{4} & 4^{3} \cdot p_{4} \\
D^{3} p_{5} & D^{2} p_{5} & D p_{5} & 5 \cdot D p_{5} & 5^{2} \cdot D p_{5} & 5^{2} \cdot p_{5} & 5^{3} \cdot p_{5} \\
D^{3} p_{6} & D^{2} p_{6} & D p_{6} & 6 \cdot D p_{6} & 6^{2} \cdot D p_{6} & 6^{2} \cdot p_{6} & 6^{3} \cdot p_{6}
\end{array}\right]
$$

$\vec{f}$ is the $7 \times 1$ column vector defined by

$$
\vec{f} \equiv\left[\begin{array}{l}
f_{1} \\
f_{2} \\
f_{3} \\
f_{4} \\
f_{5} \\
f_{6} \\
f_{7}
\end{array}\right]
$$

and $\overrightarrow{0}$ is the $6 \times 1$ column vector defined by

$$
\overrightarrow{0} \equiv\left[\begin{array}{l}
0 \\
0 \\
0 \\
0 \\
0 \\
0
\end{array}\right] \text {. }
$$

The following program, written in Mathematica 4.0 for Students and run on a Dell Dimension XPS R400 computer using Windows 98 operating system, computes the seven terms $\left\{f_{k}\right\}_{k=1}^{7}$ by setting each $f_{k}$ to the appropriate cofactor of 20 . This matrix is denoted by $T$ in the program. The symbol $s[k]$ stands for the $k$ th powersum $p_{k}$ of the roots of $P$. The output is denoted by $f$, which is defined as the transpose of $\vec{f}$. The result is

$$
\left[\begin{array}{lllllll}
-27+4 x^{3} & 18 x^{2} & 10 x & -3 x & -3 x & -3 & -1
\end{array}\right],
$$

which is the Cockle resolvent. The computation time is less than 5 seconds.

$\mathrm{x}=. ; \mathrm{s}[0]=3 ; \mathrm{s}[1]=-\mathrm{x} ; \mathrm{s}[2]=\mathrm{x}^{\wedge} 2$;

Tab1e $\left[s[k+3]=\operatorname{Expand}\left[-x^{*} s[k+2]+s[k]\right],\{k, 0,3\}\right]$;

$\mathrm{T}=\mathrm{Tab} 1 \mathrm{e}[\{\mathrm{D}[\mathrm{s}[\mathrm{k}],\{\mathrm{x}, 3\}], \mathrm{D}[\mathrm{s}[\mathrm{k}],\{\mathrm{x}, 2\}], \mathrm{D}[\mathrm{s}[\mathrm{k}], \mathrm{x}]$, $\left.\left.\mathrm{k} * \mathrm{D}[\mathrm{s}[\mathrm{k}], \mathrm{x}], \mathrm{k} \wedge 2 * \mathrm{D}[\mathrm{s}[\mathrm{k}], \mathrm{x}], \mathrm{k}{ }^{\wedge} 2 * \mathrm{~s}[\mathrm{k}], \mathrm{k} \wedge 3 * \mathrm{~s}[\mathrm{k}]\right\},\{\mathrm{k}, 1,6\}\right]$;

M=Minors $[t, 6]$;

$\mathrm{f}=$ Table $\left[\right.$ Simp 1 i fy $\left.\left[\mathrm{m}[[1, \mathrm{k}]] *(-1)^{\wedge}(7-\mathrm{k}) /(466560 * \mathrm{x})\right],\{\mathrm{k}, 1,7\}\right]$. 
To see the output in Mathematica for other variables, remove the semicolon after its formula. For the record, the first six powersums are (written in the form Mathematica gives) $p_{1}=-x, p_{2}=x^{2}, p_{3}=3-x^{3}, p_{4}=-4 x+x^{4}, p_{5}=5 x^{2}-x^{5}$, and $p_{6}=3-6 x^{3}+x^{6}$. The cofactors of the matrix $2 \mathfrak{b}$ had to be divided by $466560 \cdot x=2^{7} \cdot 3^{6} \cdot 5^{1} \cdot x$ to get the resolvent in Cohnian form, that is, such that the only divisors in $\mathbb{Z}[x, \alpha]$ among all the terms of the resolvent are \pm 1 .

\section{REFERENCES}

[1] J. Cockle, On differential equations and on co-resolvents, Transactions of the Royal Society of Victoria 7 (1865-1866), 176-191.

[2] E. R. Kolchin, Differential Algebra and Algebraic Groups, Pure and Applied Mathematics, vol. 54, Academic Press, New York, 1973.

[3] I. G. Macdonald, Symmetric Functions and Hall Polynomials, Oxford Mathematical Monographs, Clarendon Press, Oxford University Press, New York, 1995.

[4] J. M. Nahay, Linear differential resolvents, Ph.D. dissertation, Rutgers University, New Jersey, 2000.

[5] _ Powersum formula for polynomials whose distinct roots are differentially independent over constants, Int. J. Math. Math. Sci. 32 (2002), no. 12, 721-738.

[6] _ Linear relations among algebraic solutions of differential equations, J. Differential Equations 191 (2003), no. 2, 323-347.

John Michael Nahay: 25 Chestnut Hill Lane, Columbus, NJ 08022-1039, USA

E-mail address: resolvent@comcast.net 


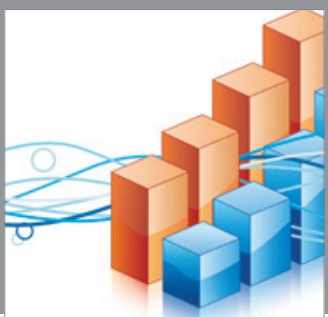

Advances in

Operations Research

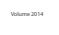

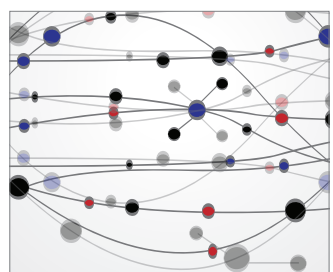

\section{The Scientific} World Journal
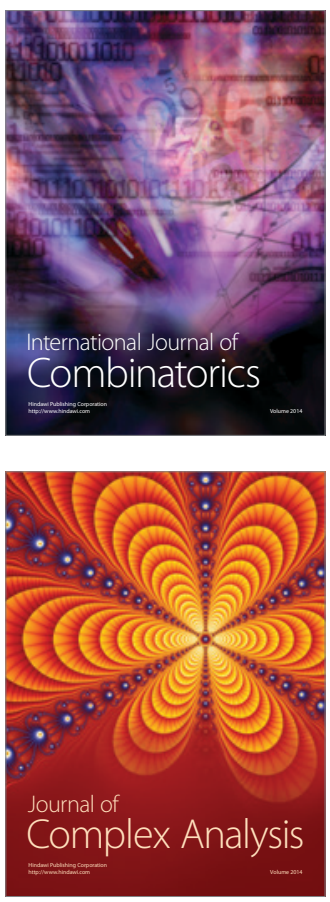

International Journal of

Mathematics and

Mathematical

Sciences
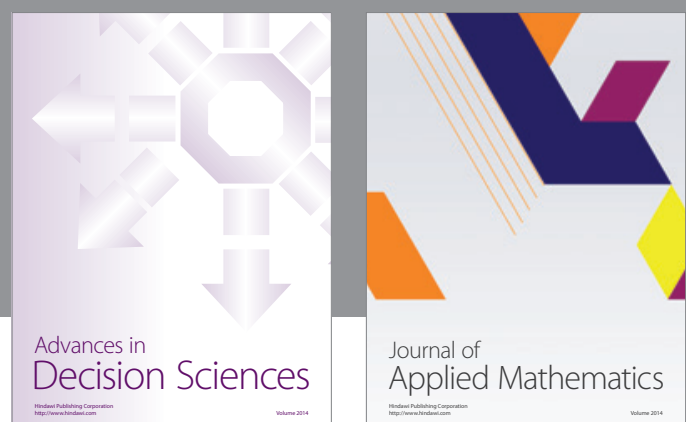

Journal of

Applied Mathematics
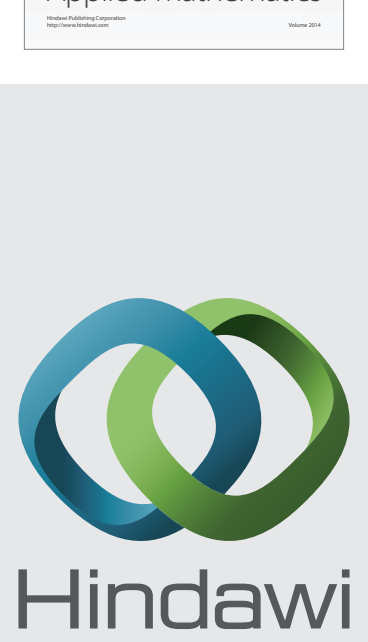

Submit your manuscripts at http://www.hindawi.com
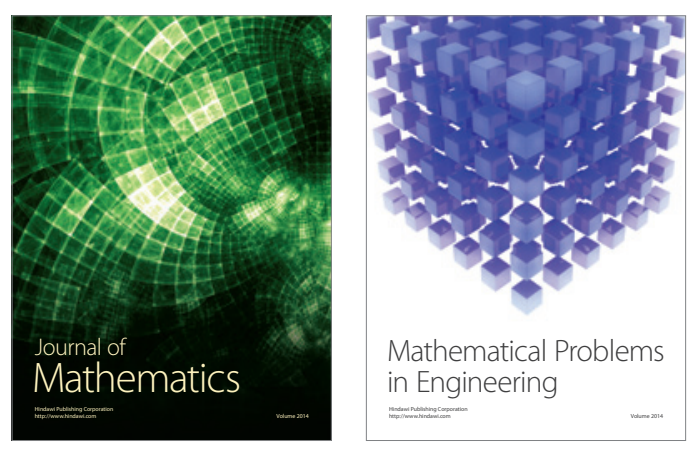

Mathematical Problems in Engineering
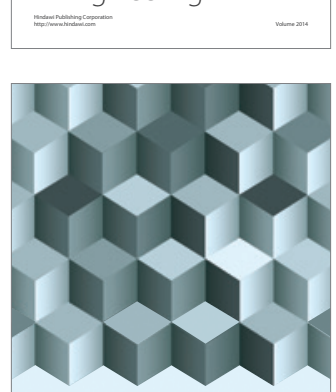

Journal of

Function Spaces
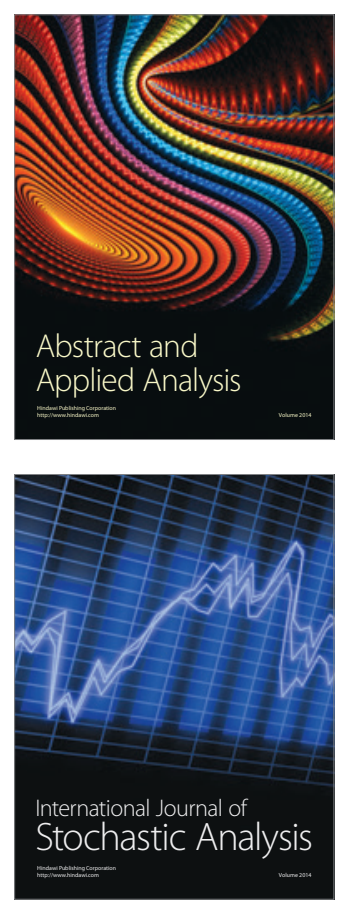

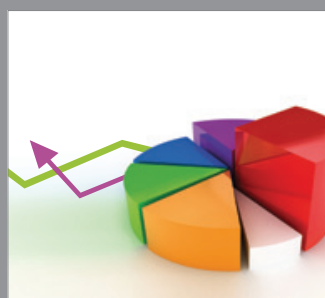

ournal of

Probability and Statistics

Promensencen
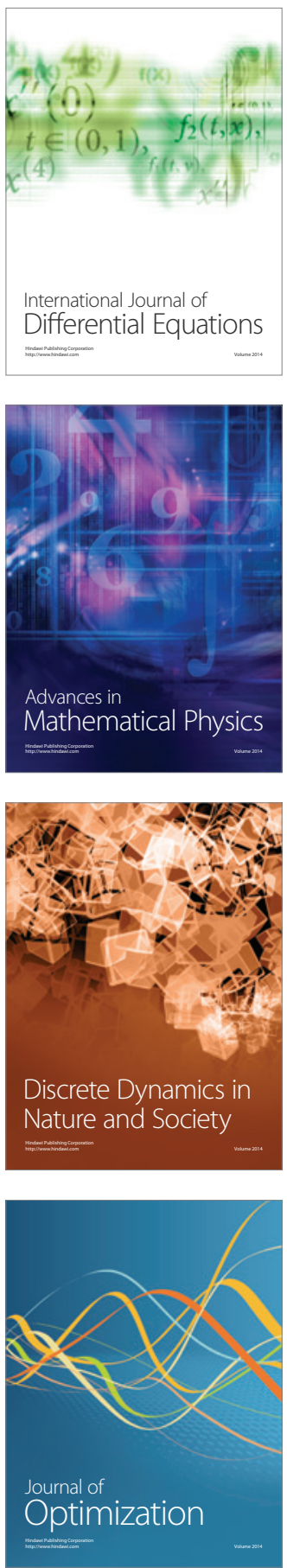